\title{
Research of Force Amplification Control Strategy of Lower Extremity Exoskeleton
}

\author{
Li Yang, Guan Xiaorong, Xu Cheng \\ School of Mechanical Engineering 105, Nanjing University of Science and Technology, Nanjing \\ 210094, China
}

Keywords: lower extremity exoskeleton; man-machine force amplification; control Strategy; joint simulation; power support effect;

\begin{abstract}
In order to gain a feasible control strategy that is appropriate for lower extremity exoskeleton, a man-machine force amplification control strategy was proposed, and the joint simulation by using Adams and Matlab/Simulink for the control strategy was carried out. It proves that the lower extremity exoskeleton with the man-machine force amplification control strategy can effectively help human body bearing heavy payload, and can reduce the energy consumption of human body in the process of walking.
\end{abstract}

\section{Introduction}

Human lower extremity exoskeleton is a kind of wearable robot that has capability of carrying a payload and can supplement human intelligence with the strength. It can be used for the soldiers to bear heavy payload when marching, so it can reduce the burden of the pilot in real time. Besides, it's capable of transporting heavy materials over rough terrain or up staircases.

At present, the control strategy is still the bottleneck of the development of lower extremity exoskeleton. The earlier lower extremity exoskeleton developed by University of Berkeley called BLEEX, H. Kazerooni $[1,2]$ et al. proposed the sensitivity amplification control algorithm for the BLEEX that can increases the closed loop system sensitivity to its pilot's forces and torques without any measurement from the pilot. But the control method has little robustness to parameter variations and therefore requires a relatively good dynamic model of the system. The lower extremity exoskeleton developed by Tomoyoshi Kawabata et al. of Tsukuba University called HAL [3], HAL produces torque corresponding to muscle contraction torque by referring to the myoelectricity that is biological information to control operator's muscles. The lower extremity exoskeleton developed by K. H. Low [4] et al. of Singapore Nanyang Technological University called NTU which uses the ZMP control strategy. But the human body dynamic walking does not meet the ZMP stability criterion [5], so the ZMP control strategy will make the pilot's walking be not very natural.

In this paper, The interaction forces of the human body with the lower extremity exoskeleton were studied, and the relationships between the interaction forces and the joint hydraulic driving forces of the lower extremity exoskeleton were analyzed, and then the man-machine force amplification control strategy which aimed at minimizing the man-machine interaction forces and reducing the energy consumptions of human body when carrying a payload to walk was proposed. And the power support effect of the exoskeleton was analyzed by using the dynamics simulation software Adams and Matlab/Simulink for joint simulation, and then the feasibility of this control strategy was verified.

\section{Force Amplification Control Strategy}

Structural Model. The coordinate system usually used for the movement of human body is as follows: the $\mathrm{X}$ axis is toward the front of human body, and the $\mathrm{Z}$ axis is vertical upward, and the $\mathrm{Y}$ axis can be determined with the right hand rule. Generally, the $\mathrm{Y}$ axis is commonly referred to as flexion/extension axis; the $\mathrm{X}$ axis is commonly referred to as adduction/abduction axis; and the $\mathrm{Z}$ axis is commonly referred to as rotation axis [6]. 
Similar to most researchers, the human lower extremity exoskeleton model is designed by using humanoid design in this paper. The hip joint has degrees of freedom on flexion/extension axis and adduction/abduction axis, and different from the degree of freedom on flexion/extension axis that is actuated by hydraulic cylinder the degree of freedom on adduction/abduction axis is in passive state; the knee joint only has an actuated degree of freedom on flexion/extension axis; the ankle joint has all the three rotational degrees of freedom, and the three degrees of freedom are in passive state.

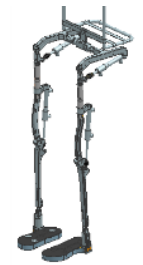

Fig. 1: 3D model of the lower extremity exoskeleton

Control Model. It will produce a man-machine interaction force when the movement of human body doesn't match completely with the lower extremity exoskeleton, and the goal of the control strategy of lower extremity exoskeleton is to reduce the energy consumption of human body by minimizing the interaction force. So in the process of movement, the driving forces of hydraulic cylinders of exoskeleton are obtained by detecting and enlarging the interacting forces, and then the man-machine interaction forces will have decreasing trend when the driving forces are applied to the exoskeleton.

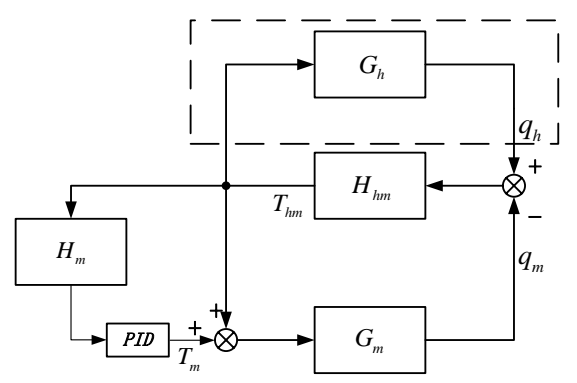

Fig.2: Block diagram of the control model

In the block diagram: $q_{h}$ denotes the angle of each human body joint; $q_{m}$ denotes the angle of each lower extremity exoskeleton joint; $T_{h m}$ denotes the torque applied to each lower extremity exoskeleton joint by human body; $T_{m}$ denotes the torque applied to each lower extremity exoskeleton joint by drive system; $G_{h}$ denotes the transfer function that human body driving each joint of itself, and it does not need to be specified when using the control strategy; the transfer function $H_{h m}$ denotes the relationship of man-machine interaction force and exoskeleton joint torque, the transfer function $G_{m}$ denotes the relationship of exoskeleton joint torque and exoskeleton joint angle, and the two transfer functions are the system's own characteristics, they also do not need to be specified. The only element which needs to be determinate is the transfer function $H_{m}$ of enlarging the man-machine interaction force to the hydraulic driving force.

This control strategy is a kind of pure force control; because the lower extremity exoskeleton is worn on the body, the motion control of the lower extremity exoskeleton will be implemented by the pilot. This method does not need to use myoelectricity sensors, and also does not need to establish an accurate mathematical dynamic model. Besides, the lower extremity exoskeleton can also follow to the human body very well.

\section{Establishment of the Simulation Model}

Multi-body Dynamics Simulation Model. Different simulation cases were combined to compare for the auxiliary effect of the lower extremity exoskeleton: the man-machine coupled model of human body, lower extremity exoskeleton and payload; 2 the case of human body directly bearing payload; 3 the case of human body without bearing payload. 


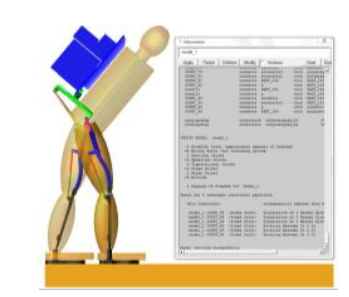

Fig. 3: Man-machine coupled model

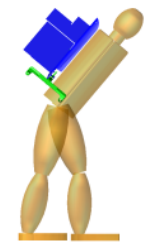

Fig.4: Case of human body directly bearing payload

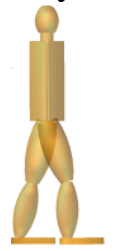

Fig.5: Case of human body without bearing payload

When simulating, all degrees of freedom on human body joints are actuated by the data curve (the curve of angle changing with time) that are obtained in the form of independently collected CGA (Clinical Gait Analysis) data, and only the four set of hydraulic cylinders of the lower extremity exoskeleton are actuated by pressure curve, all other degrees of freedom are in passive state. In addition, the pilot and the lower extremity exoskeleton have rigid mechanical connections at the torso and feet.

Control Strategy Simulation Model. The control strategy simulation model is shown in figure 5.

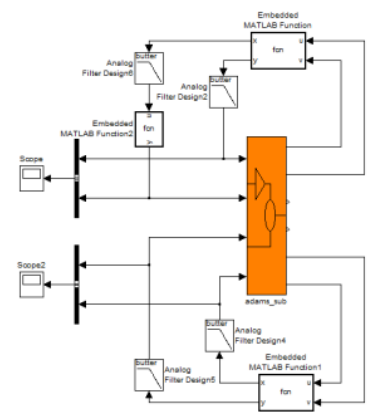

Fig.6: Control strategy simulation model

The adams_sub module is the interaction module of Adams with Matlab/Simulink. Because only the process that the right leg is in stance configuration and the left leg is in swing configuration is simulated, so the interaction force of right foot is not used for the time being.

This control strategy simulation model can form a loop. Firstly, the interaction forces of the human body and foot with the lower extremity exoskeleton are detected from the multi-body dynamics model in Adams in real-time. And then the interaction forces are outputted to Matlab/Simulink as input parameters of the control strategy. After that, the driving forces of the exoskeleton hydraulic cylinders are obtained through certain amplifier and filter process in Matlab/Simulink, and then they are outputted to Adams.

\section{Compare of Different Simulation Cases.}

Simulation of Man-machine Coupled Model. In sport biomechanics, human body model can be simplified as multi-rigid-body system consisting of multiple rigid links [7]. The kinematic and dynamic parameters of human body like angle, torque, power and so on can be obtained by doing the joint simulation of Adams with Matlab/Simulink. 
The simulated whole moving process is as shown in figure 7.

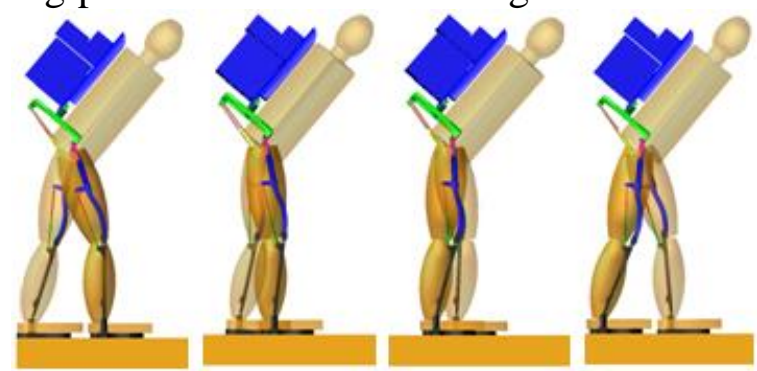

Fig. 7. The simulated whole moving process

Drive Curves of the Four Hydraulic Cylinders. The pressure curves for the four hydraulic cylinders of the lower extremity exoskeleton that calculated by the control strategy of the simulation model are shown in figure 8.

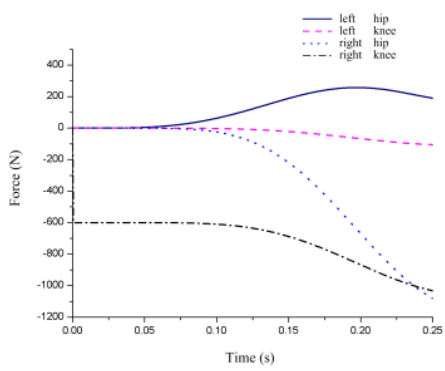

Fig.8: Pressure curve of the four hydraulic cylinders

In the whole process, the right leg is in stance configuration and the left leg is in swing configuration. It can be seen from the figure 6 that the biggest pressure of the hydraulic cylinders is approximately equal to the sum of the weight of the payload $(100 \mathrm{~kg})$ and the lower extremity exoskeleton $(10 \mathrm{~kg})$. It can also be seen that the pressures of the hydraulic cylinders of right leg are larger than that of left leg, because the whole payload force is transferred to the ground by the stance leg. Besides, the needed pressure of the hydraulic cylinders of left hip joint is much larger than that of left knee joint, because the left hip needs to swing the whole leg other than the left knee just needs to swing the shank.

Driving Power of Human Joints. The driving power of each human joints of the whole process is shown in figure 9 and figure 10.
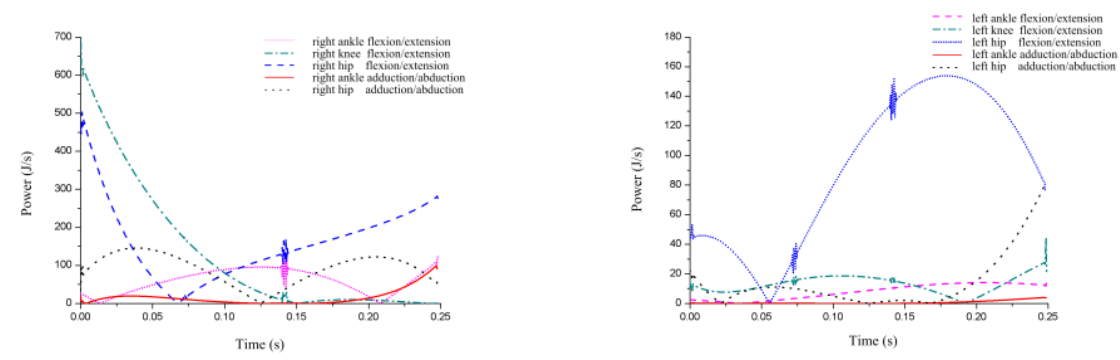

Figure 9.Driving powers of right joints Figure 10.Driving powers of left joints

Similar to the pressure curves, it can be seen that the driving power of right leg joints of human body is bigger than left leg joints. But the driving powers of hip joint are bigger than other joints in both the two human legs.

Compare of Energy Consumptions of Different Simulation Cases. The energy consumption in the whole process of each degree of freedom of human body can be calculated by integrating the power curve.

Table 1 Energy consumption of each joint of different cases

\begin{tabular}{c|cc|cc|cc}
\hline Case & \multicolumn{2}{c}{ I } & \multicolumn{2}{c}{ II } & \multicolumn{2}{c}{ III } \\
\hline Degree of freedom & fl/ex & ad/ab & fl/ex & ad/ab & fl/ex & ad/ab \\
\hline Energy of right ankle $(J)$ & 11.9 & 1.7 & 14.6 & 3.7 & 18.1 & 4.4
\end{tabular}




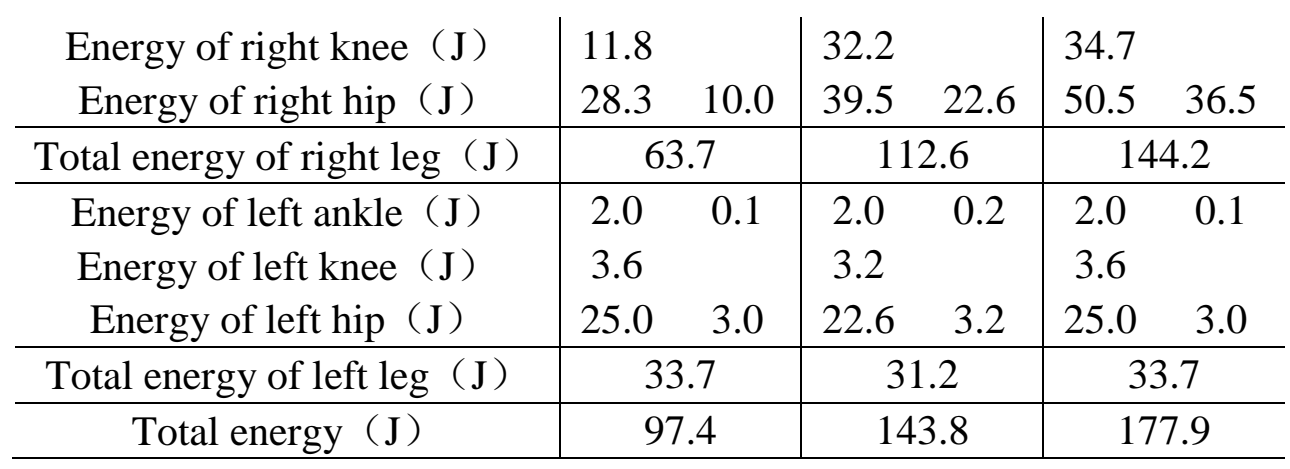

Notes: fle/ext means flexion/extension; add/abd means adduction/abduction; I: Human body; II: Human body+ lower extremity exoskeleton+ payload; III: Human body + payload

According to statistics, it founds that the power consumption of $63 \mathrm{~kg}$ male is about $3 \mathrm{kcal} / \mathrm{min}$ when walking at $1.33 \mathrm{~m} / \mathrm{s}$ speed; $6 \mathrm{Kcal} / \mathrm{min}$ at $1.77 \mathrm{~m} / \mathrm{s}$ speed; $10 \mathrm{Kcal} / \mathrm{min}$ at $2.7 \mathrm{~m} / \mathrm{s} \mathrm{speed}$. Walking at $1.51 \mathrm{~m} / \mathrm{s}$ speed belongs to the normal speed walking, fewer muscles are mobilized. Energy is mainly used to adjust the body center of gravity, and the energy consumption in the walking process is focused on the leg [8].The established human body model weighs about $70 \mathrm{~kg}$ without arms. It can be calculated out from the table that the total energy consumption of the human legs in the walking process is $(63.7+33.7) \mathrm{J} / 0.25 \mathrm{~s}=5.7 \mathrm{Kcal} / \mathrm{min}$, which is coincident with the results of statistics.

It can be seen that the energy consumption of right leg of case II is much lower than case III, and the energy consumption of left leg of case II is even slightly lower than case I. The total energy consumption of model III is $80.5 \mathrm{~J}$ more than model I, and the model II is $46.4 \mathrm{~J}$ more than model I, so the lower extremity exoskeleton can reduce (80.5-46.4) $/ 80.5=42.4 \%$ of the total energy consumption of human body for extra bearing $100 \mathrm{Kg}$ payload. The energy consumption of human body can be further reduced by optimizing the man-machine interaction force amplification transfer function $\mathrm{Hm}$.

From what has been discussed above, the designed lower extremity exoskeleton and the control strategy can provide the ability to carry significant payloads with minimal effort to its operator.

\section{Conclusion}

In order to gain a feasible control strategy that is appropriate for lower extremity exoskeleton, the man-machine force amplification control strategy was proposed. The structure model and control model of the lower extremity exoskeleton was established, and then the joint simulation model between Adams and Matlab/Simulink was set up. Based on the results of the joint simulation of control strategy model and multi-body dynamics simulation model, the power support effects of different cases were compared and analyzed. It can be seen that the energy consumption can obviously reflect the power support effect of lower extremity exoskeleton, because the designed lower extremity exoskeleton and the control strategy can reduce about $42.4 \%$ of the total energy consumption of human body for extra bearing $100 \mathrm{Kg}$ payload in the process of walking. It proves that the lower extremity exoskeleton with the man-machine force amplification control strategy can effectively help human body to bear heavy payload, and can achieve the goal of reducing the burden of human body in the process of walking.

\section{References}

[1] Ryan Steger, Sung Hoon Kim. Control Scheme and Networked Control Architecture for the Berkeley Lower Extremity Exoskeleton (BLEEX) [C]. IEEE International Conference on Robotics and Automation, May 2006, Florida.

[2] Yang Zhiyong, Gu Wenjing, Zhang Jing, Gui Lihua. Force Control Theory and Method of Soldier Load Carrying Exoskeleton Suit [M]. National defense industry press, 2013, 11.

[3] Tomoyoshi Kawabata, Hozumi Satoh. Working Posture Control of Robot Suit HAL for 
Reducing Structural Stress [C]. IEEE International Conference on Robotics and Biomimetics, December 19 -23, 2009, Guilin, China.

[4] K. H. Low, Xiaopeng Liu, Haoyong Yu. Development of NTU Wearable Exoskeleton System for Assistive Technologies [C]. IEEE International Conference on Mechatronics \& Automation Niagara Falls, July 2005, Canada.

[5] Fu Chenglong. Section Mapping Stability Criterion and Application of the Plane Biped Robot [D]. Beijing: tsinghua university Ph.D. Thesis, 2006.

[6] Adam Zoss, H.Kazerooni, Andrew Chu. On the Mechanical Design of the Berkeley Lower Extremity Exoskeleton (BLEEX) [C]. IEEE International Conference on Robotics and Automation, April 2005, Barcelona.

[7] The national sports colleges and universities teaching committee. Sports Biomechanics [M]. People's sport publishing house, 2009:1-2.

[8] Wang Huan, Jiang Chong-min, Liu Xin. Walking Energy Expenditure and Recommendation in Chinese Adults [J]. China Sport Science, 2013, 33 (11): 89-93. 